\title{
Computer-Generated Nuclear Features Compared With Axillary Lymph Node Status and Tumor Size as Indicators of Breast Cancer Survival
}

\author{
WILLIAM H. WOLBERG, MD AND W. NICK STREET, PHD
}

\begin{abstract}
The extent to which malignant cells deviate from normal is generally accepted to be a prognostic indicator. However, assessing the degree of deviation has been subjective and poorly reproducible. Our goal is to develop a computer program for objectively measuring nuclear size, shape, and texture from histologic slides and to make the program available on the Internet. We used this program to analyze 353 histologic sections obtained from patients with invasive breast cancer who were diagnosed and treated from 1981 through 1995 and who had determinable outcomes. The median follow-up was 8.3 years. We compared the relationship of survival with our computer-derived nuclear features versus axillary lymph node status and tumor size. We believe that our results are generally applicable because our patient survival, when stratified by lymph node status, was similar to that of the 24,000 breast cancer patients in the National Cancer Institute's Surveillance, Epidemiology, and End Results pro-
\end{abstract}

In this study we examined the relationship between breast cancer survival, the classical prognostic criteria of tumor size and lymph node status, ${ }^{1}$ and computer-generated nuclear features in histologic sections. We used a semiautomatic program that we developed $^{2}$ and call Xcyt (available free of charge at http:/ / dollar.biz.uiowa.edu/ street/xcyt/xcyt.html). No special tissue processing is required; thus others can verify our results with their own samples.

Previously, we used Xcyt to analyze fine-needle aspirates of breast cancers. In those cytologic samples, the nuclear feature of largest perimeter was prognostically stronger than either lymph node status or tumor size. ${ }^{3}$

\section{MATERIALS AND METHODS}

\section{Patients}

Six hundred and twenty-nine patients with invasive breast cancer and without distant metastases underwent surgery from April 1981 through 1995. All surgery was done at the University of Wisconsin Hospital, and all cases were diagnosed by a University pathologist and reviewed according to

From the Department of Surgery, University of Wisconsin, Madison, WI and the Management Sciences Department, University of Iowa, Iowa City, IA. Accepted for publication July 11, 2002.

Supported in part by National Science Foundation grant IIS-9996044.

Address correspondence and reprint requests to William $\mathrm{H}$. Wolberg, MD, Department of Surgery, University of Wisconsin, 600 Highland Ave., Madison, WI 53792.

Copyright 2002, Elsevier Science (USA). All rights reserved.

0046-8177/02/3311-0005\$35.00/0

doi:10.1053/hupa.2002.129198 gram. In multivariate analysis, the strongest prognostic factor was the largest nuclear area, followed by tumor size and the extent of axillary lymph node involvement. The mean area of the 3 largest nuclei when combined with tumor size identified $30 \%$ of all breast cancer patients who had an $87 \%$ 15-year breast cancer-specific survival. Inclusion of lymph node status added little to this 2-factor model. Routine axillary lymph node surgery for prognostic purposes may become unnecessary, because nuclear features may provide sufficient information. Hum Pathol 33:1086-1091. Copyright 2002, Elsevier Science (USA). All rights reserved.

Key words: breast cancer, prognosis, image analysis, computer analysis, telemedicine.

Abbreviations: $\mathrm{N}-$, node negative; $\mathrm{N}+$, node positive; SEER, Surveillance, Epidemiology, and End Results; TNM, tumor size, lymph node, metastasis.

quality assurance standards. Three hundred and fifty-three cases had determinable outcomes and tumor tissue available for resectioning. In each case, the histologic diagnosis was the one originally made. The diagnostic blocks were retrieved and recut. Most of the patients excluded from the study had undergone excisional biopsy at another facility but had axillary surgery with negative nodes at our institution. Material was not available for resectioning in such patients. For this reason, only 153 of the 333 node-negative $(\mathrm{N}-)$ patients could be included in this study, compared with 200 of the 262 node-positive $(\mathrm{N}+)$ patients. The mean tumor size was $2.7 \mathrm{~cm}$ for patients unavailable for follow-up, compared with $2.8 \mathrm{~cm}$ for those included in the study. For study patients, the median follow up was 8.3 years; 23 patients were followed for at least 15 years. Three hundred and thirty-seven patients underwent axillary lymph node dissections and pathologic staging. The 16 patients who did not undergo axillary surgery were clinically $\mathrm{N}-$ and had either small tumors of favorable histology or complicating medical conditions. None of the clinically staged patients subsequently developed axillary recurrences or distant metastases.

Our patient population is representative of the general breast cancer patient population of the United States. When stratified by axillary lymph node involvement, our patients' survival was similar to that of 24,000 similar patients from the the National Cancer Institute's Surveillance, Epidemiology, and End Results (SEER) program. ${ }^{4}$ Survival was superimposed for the first 5 years and deviated only slightly thereafter (Fig 1 ). The differences between 5 and 10 years is not surprising because of the small number of our patients who had positive nodes and survived beyond 5 years and because of the decreasing prognostic importance of lymph node status with time. .-7 $^{5-1}$

\section{Sample Preparation}

Tissues were fixed in $10 \%$ neutral formalin, embedded in paraffin, and cut into $6-\mu$ sections. These sections were stained with Weigart's hematoxylin without counterstaining. 
FIGURE 1. Breast cancer-specific survival of our patients (thin black lines) compared with that of the SEER program (wide gray lines), when stratified according to axillary lymph node involvement at 0 (NO), 1 to 3 (N1-3), and 4 or more positive nodes $(\mathrm{N}>3)$.

\section{Selection of Nuclei for Analysis}

A single, maximally atypical field was visually selected for analysis. A $640 \times 480$ digital image of the field was made using a $\times 63$ objective on a microscope with a $\times 2.5$ ocular on the video camera port. A frame grabber board (WinVision Pro; Quanta, Mountain View, CA) was used to capture the field as a .gif image. At this magnification, 3.9 pixels equal 1 micron in 1 dimension and 15.21 pixels equal 1 square micron in 2 dimensions. Analysis was performed on 10 to 100 nuclei per patient, depending on the number of nuclei present in the field.

\section{Automatic Identification of Nuclei}

The Xcyt program automatically locates and outlines cell nuclei in an image by searching for objects that match 1 of a collection of elliptical templates. The templates are updated as the system is used to reflect the shape of the objects found in previous images. In this way, the search becomes both faster and more accurate as the system is used. (Street ${ }^{8}$ has provided more details on the image analysis performed by Xcyt.) On average, approximately $75 \%$ of the nuclei in the images are correctly outlined. Erroneous outlines are edited or deleted by the user, and missed nuclei are traced manually.

\section{Nuclear Morphometric Features}

The following nuclear features were computed for each identified nucleus ${ }^{2}$ :

- Radius was computed by averaging the length of radial line segments from the center of the nuclear mass to each of the points of the nuclear border.

- Perimeter was measured as the distance around the nuclear border.

- Area was calculated by counting the number of pixels in the interior of the nuclear border, then adding half of the pixels on the perimeter.

- Perimeter and area were combined to give a measure of the compactness of the cell nuclei using the formula perimeter ${ }^{2} /$ area.

- Smoothness was quantified by measuring the difference between the length of each radius and the mean length of adjacent radii.

- Concavity measured the size of any indentations in the nuclear border.

- Concave points counted the number of points on the nuclear border that lie on an indentation.

- Symmetry was measured by finding the relative difference in length between line segments perpendicular to and on either side of the major axis.

- Fractal dimension was approximated using the "coastline approximation" described by Mandelbrot, ${ }^{9}$ which measures nuclear border irregularity.

- Texture was measured by finding the variance of the gray scale intensities in the component pixels.

For these 10 features, the computer calculated the mean value, the "largest" value, and the standard error for each nuclear feature, resulting in a total of 30 features. The largest value for each feature was the mean of the 3 largest values for all nuclei in the analyzed image; 3 was chosen as the smallest number that would guard against numerical instability in the shape features.

\section{Statistical Analysis}

Calculations were done using SPSS software (SPSS, Chicago, IL). ${ }^{10,11}$ The first step was to use the Cox univariate analysis to rank the available predictive factors according to their individual ability to predict time of breast cancer death. The independent variables were the 30 nuclear features, along with tumor size and lymph node status. Patients were then assigned to groups based on splits for each pair of the 3 strongest prognostic factors: tumor size, largest nuclear area, and lymph node positivity. Splits were at the median for tumor size and largest area and positive or negative for axillary lymph node status. 
TABLE 1. Comparison of Prognosis for Patients Divided Into Groups Based on Largest Nuclear Area of Their Cancers

\begin{tabular}{ccc}
$\begin{array}{c}\text { Nuclear Largest Area } \\
\text { Cutpoint }\left(\mu^{2}\right)\end{array}$ & $P$ & $\begin{array}{c}\text { Relative Risk } \\
\text { Factor }\end{array}$ \\
\hline 45.6 & .0200 & 3.9 \\
58.4 & .0009 & 4.1 \\
67.6 & .0007 & 2.7 \\
78.0 & .0004 & 2.4 \\
87.1 & .0757 & 1.8 \\
100.5 & .0004 & 2.1 \\
114.9 & .0002 & 2.2 \\
137.0 & .0004 & 2.3 \\
172.3 & .0094 & 2.1 \\
\hline
\end{tabular}

NOTE. Cox regression analysis of breast cancer survival was performed for patients with cancers with larger nuclear largest areas than the cutpoints listed here compared with patients with having smaller ones.

\section{RESULTS}

The following nuclear features were highly related to breast cancer-specific overall survival in univariate analysis $(P<.0001)$ : mean area, mean perimeter, mean radius, standard error of area, largest area, and largest perimeter. Nuclear features of lesser significance $(P$ between .0001 and .01) were mean fractal dimension, standard error of perimeter, standard error of radius, and largest texture. Additionally, the number of cancerous axillary lymph nodes, and tumor size were highly significant with $P<.0001$. Overall, the 3 most significant factors were tumor size, axillary lymph nodes, and largest nuclear area. In a 3-factor Cox multivariate analysis model, the significance of largest area was $P<.0001$, tumor size was $P=.0001$, and $\mathrm{N}+$ or $\mathrm{N}-$ was $P=.0171$. To determine the robustness of largest area as a prognostic factor, the patients were divided into deciles according to largest area. At all deciles, patients whose tumors had larger largest area nuclei had a poorer prognosis than those with smaller ones (Table 1). In comparison, $\mathrm{N}+$ versus $\mathrm{N}-$ was $P=.0002$ with a relative risk of 2.5 , and tumor size $\geq 2.4 \mathrm{~cm}$ versus tumor size $<2.4 \mathrm{~cm}$ was $P<.0001$ with a relative risk of 3.8. We used the median cutpoint for largest area, even though the poorest prognostic separation occurred at the median cutpoint. Other cutpoints may produce better results.

Patients were divided according to the median values for largest area $\left[87.1 \mu^{2}(1325\right.$ pixels $\left.)\right]$, for tumor size $(2.4 \mathrm{~cm})$, and according to $\mathrm{N}+$ or $\mathrm{N}-$ status. At these cutpoints, Kaplan-Meier survival analysis showed that patients were best separated according to whether their tumor size was smaller or larger than the median size, next best separated according to whether they were $\mathrm{N}+$ or $\mathrm{N}-$, and least well separated by whether their tumor's nuclear largest area was smaller or larger than the median large area. The log rank statistics were $32.8(P<.0001), 15.2(P=.0001)$, and $7.9(P=.0049)$, respectively. These 3 features were then paired according to the aforementioned splits to create 4 groups of patients for each pair. Taken in pairs, the overall $\chi^{2}$ value in Cox multivariate models was 50.0 for tumor size and largest area, 47.2 for largest area and lymph nodes, and 42.3 for tumor size and lymph nodes. Because the pair of tumor size and largest area gave the best separation, further studies were done with this pair; specifically, size $<2.4 \mathrm{~cm}$ and largest area $<87.1 \mu^{2}$ (107 patients), size $<2.4 \mathrm{~cm}$ and largest area $\geq 87.1 \mu^{2}$ (67 patients), size $\geq 2.4 \mathrm{~cm}$ and largest area $<87.1 \mu^{2}$ (67 patients), and size $\geq 2.4 \mathrm{~cm}$ and largest area $\geq 87.1 \mu^{2}$ (106 patients). Within these 4 groups, Kaplan-Meier analysis ${ }^{12}$ revealed no significant difference between breast cancer survival of $\mathrm{N}+$ and $\mathrm{N}-$ patients, and no significant differences were noted in the number of patients with distant disease recurrence and in breast cancer survival (Student's $t$ test). The recurrences of distant disease in these 4 groups are displayed in Figure 2.

The 15-year breast cancer-specific survival for these 4 groups was $86.6 \%, 77.9 \%, 35.4 \%$, and $48.9 \%$, respectively. All group separations were significant except when the size of the cancer was $\geq 2.4 \mathrm{~cm}$, in which case the nuclear largest area was insignificant (Table 2). Kaplan-Meier survival curves ${ }^{12}$ are shown in Figure 3, and the significance of the difference between the curves is delineated in Table 2.

\section{DISCUSSION}

Several researchers, beginning with Black et al, ${ }^{13,14}$ showed that cellular features were related to outcome. Subsequently, various grading systems were proposed. However, nuclear grade is rarely used as a prognostic indicator because of the subjectivity inherent in its assessment. ${ }^{15}$ Instead, decisions regarding postopera-

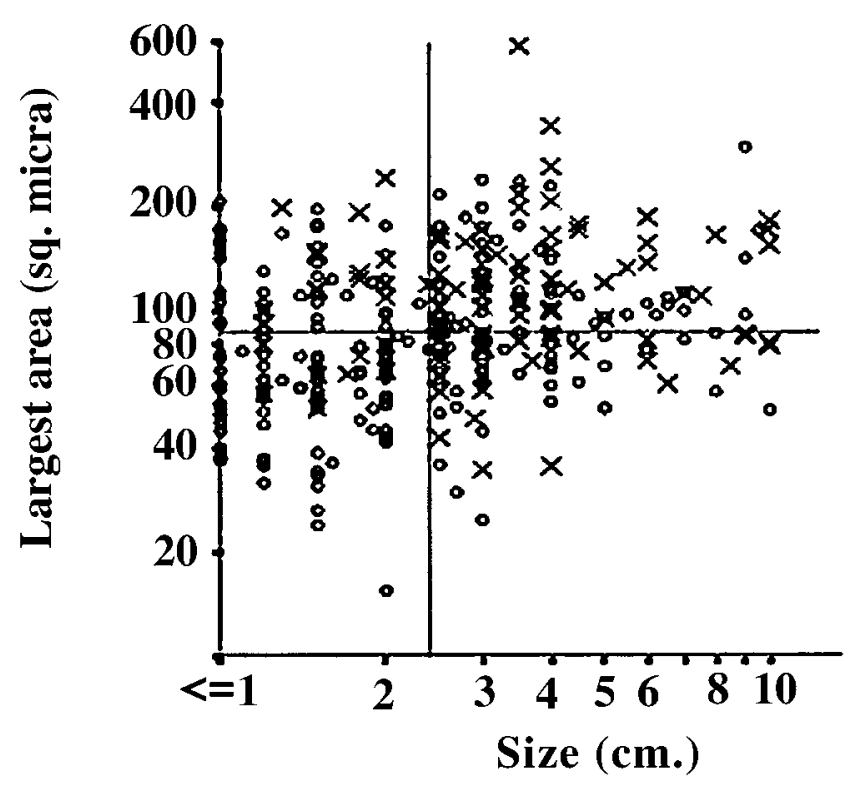

FIGURE 2. Individual values for patients with recurring $(X)$ or not recurring $(\bigcirc)$ breast cancer relative to the median value cutpoints for tumor size and largest area. Note the log-log graph because of the predominance of smaller tumors and smaller nuclear largest area. 
TABLE 2. Ten-Year Breast Cancer-Specific Survival Values and the Significance Between the Kaplan-Meier Curves

\begin{tabular}{ccc}
\hline & & Log Rank Differences Between Groups $(P)$ \\
\cline { 2 - 3 } Group & $\begin{array}{c}\text { Small Size and Small } \\
\text { Largest Area }\end{array}$ & $\begin{array}{c}\text { Large Size and Small } \\
\text { Largest Area }\end{array}$ \\
\hline $\begin{array}{l}\text { Large size and small largest area } \\
\text { Small size and large largest area }\end{array}$ & $<.0001$ & .0252 \\
Large size and large largest area & $<.0001$ & .0252 \\
\hline
\end{tabular}

NOTE. Small size, tumor size $<2.4 \mathrm{~cm}$; large size, tumor size $\geq 2.4 \mathrm{~cm}$; small largest area, nuclear feature of largest area $<87.1 \mu^{2}$; large largest area, nuclear feature of largest area $\geq 87.1 \mu^{2}$.

tive treatment regimens are typically based primarily on the tumor size, lymph node, metastasis (TNM) staging system. ${ }^{16}$ Determination of axillary node status for TNM staging necessitates surgical removal of axillary lymph nodes. Complete removal leaves the patient with numbness and other complications, including a $15 \%$ risk of arm lymphedema. ${ }^{17}$ Less- extensive dissections, such as sentinel lymph node sampling, ${ }^{18,19}$ are promising but not yet clinically established approaches. Thus the focus of our research has been on investigating the prognostic significance of objectively defined nuclear features and to determine whether the breast cancer staging without using lymph node information is feasible.

Other computer-based studies have demonstrated that larger nuclear size portends a poorer prognosis. ${ }^{20-24}$ Our largest area values were about $70 \%$ greater than the mean area values $\left(101 \pm 56 \mu^{2}\right.$ versus $60 \pm 28$ $\mu^{2}$ ) and probably identified multiploid nuclei that have

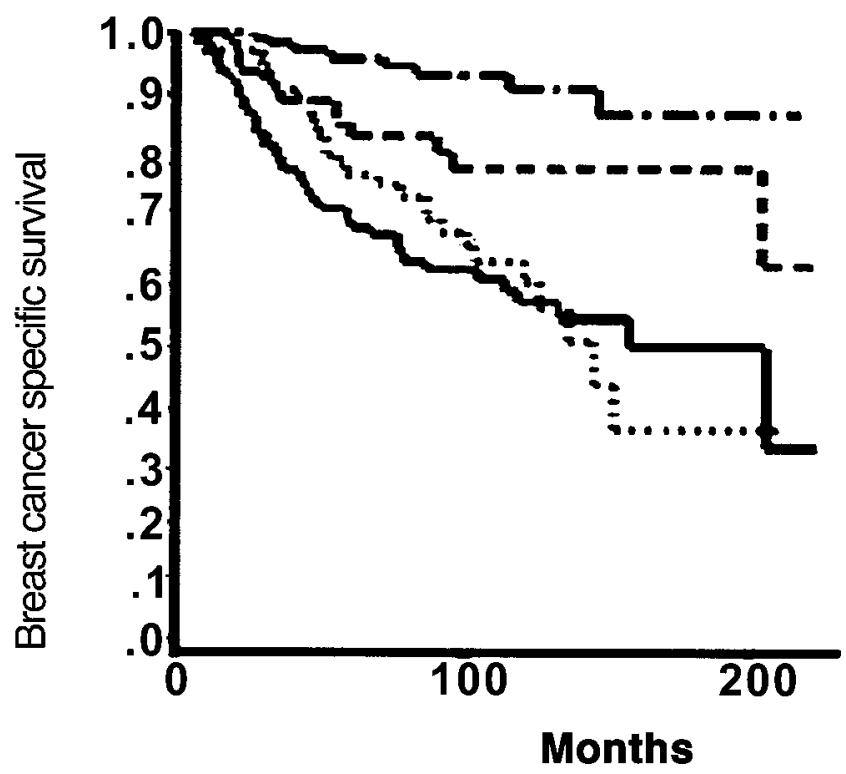

FIGURE 3. Kaplan-Meier curves for breast cancer-specific survival for the 4 groups determined by the median cutpoints for tumor size and nuclear largest area. $\cdot-\cdot-$, size $<2.4 \mathrm{~cm}$ and largest area $<87.1 \mu^{2}$ (107 patients); - - - - , size $<2.4 \mathrm{~cm}$ and largest area $\geq 87.1 \mu^{2}$ (67 patients); $\cdots$, size $\geq 2.4 \mathrm{~cm}$ and largest area $<87.1 \mu^{2}$ (67 patients) $\cdot ;-$, size $\geq 2.4 \mathrm{~cm}$ and largest area $\geq 87.1 \mu^{2}$ (106 patients). been shown by both flow cytometry and DNA labeling index to be associated with an unfavorable prognosis. $25-29$

In our patients, tumor size was a stronger prognostic feature than axillary lymph node status, yet other studies found lymph node status to be the stronger feature. ${ }^{30}$ Our patient population differed from older series, because our study included small cancers that were identified either by either aggressive fine-needle aspiration or mammography. For instance, $2.54 \%$ of our patients had tumors smaller than $0.5 \mathrm{~cm}$ compared with $0.78 \%$ of patients in the SEER database. We found that $\mathrm{N}+$ versus $\mathrm{N}-$ was the second to tumor size in strength as a prognostic factor, yet largest area was stronger in multivariate analysis. This is explained because tumor size and $\mathrm{N}+$ or $\mathrm{N}-$ are closer correlated than are tumor size and large area (Pearson correlation coefficient of 0.459 versus 0.199 ). Therefore, largest area adds more information to tumor size than does axillary lymph node status. Within the 4 groups based on tumor size and largest area, we were unable to demonstrate significant breast cancer survival differences between $\mathrm{N}+$ and $\mathrm{N}-$ patients. Yet nodal positivity was statistically significant $(P=0.0171)$ in the 3-factor Cox model. Further studies with more patients are needed to determine whether additional clinically useful prognostic information can be obtained from axillary lymph node examination.

In previous cytologic analyses of breast fine-needle aspirates, ${ }^{3}$ we found that the nuclear size feature of largest perimeter was the strongest prognostic nuclear feature for overall survival. This is consistent with our current results, because largest perimeter and largest area are strongly correlated (Pearson correlation coefficient $.957, P<.001$ ). The only inconsistency between our cytologic and histologic findings lies in the relative importance of nuclear size and tumor size above the median cutpoints. Cytologically, large and small-sized cancers had a similarly poor prognosis when associated with unfavorable cytology. Histologically, favorable or unfavorable cytology was important only in small-sized cancers and made no difference in larger ones. To try to understand this inconsistency, we compared cytology and histology in the 136 patients for whom both types of material was available. Surprisingly, the mean values for largest perimeter and for largest area were virtually identical in both types of preparations. The correlation 
(Pearson) between cytologic largest perimeter and histologic largest area was $0.54(P<.001)$. Although highly statistically significant, there were sufficient differences in individual patients to explain the inconsistency between cytologic and histologic results.

Our study's endpoint was breast cancer-specific survival rather than either distant disease-free survival or overall survival for 3 reasons. First, our study was not controlled for the use of adjuvant chemotherapy. Axillary lymph node-positive patients were generally given adjunctive chemotherapy, whereas $\mathrm{N}-$ patients were not. The protocol adjunctive chemotherapy, mainly cyclophosphamide, methotrexate, 5-fluorourcil (CMF)based, that was given to our $\mathrm{N}+$ patients was shown to prolong distant disease-free survival but to not increase breast cancer-specific survival. ${ }^{31-33}$ Second, death from causes unrelated to breast cancer was common because of the long follow-up and the preponderance of elderly patients. Third, the time of recurrence was difficult to document during long-term follow-up because patients were followed at inconsistent intervals after 5 years. Nonetheless, similar results were obtained when distant disease- free survival rather than overall breast cancerspecific survival was used as the endpoint.

Only modest time and money investments are required to add Xcyt to a pathologist's practice. Sample preparation is similar to that used for routine histologic examination, except that Weigart's hematoxylin is used to accentuate nuclear membranes and no counterstain is applied. Images can be captured with either a video or digital still camera mounted on a microscope. In many instances, an existing 35-mm film camera can be replaced by a digital single-lens reflex camera, and the acquired image files can be transferred to a computer without a video camera or a frame-grabber board. A maximally atypical field can be selected for analysis during the course of routine histologic examination. The Xcyt analysis takes about 15 minutes. Xcyt automatically outlines the first 5 nuclei relatively slowly but speeds up markedly for subsequent nuclei. After automatic outlining, an operator edits errant outlines and manually outlines missed nuclei. Nuclear feature calculations are virtually instantaneous.

We conclude that the prognostic importance of tumor size and axillary lymph node status needs to be evaluated in conjunction with computer-based image analysis. Currently, the major reason for axillary lymph node staging is to determine the need for adjunctive chemotherapy and the type of this therapy indicated. Tumor size together with the information obtained via image analysis may provide comparable prognostic information and thus obviate the need for routine axillary lymph node surgery.

Acknowledgement. The authors are indebted to all of those who have contributed to the success of the Xcyt project, including Olvi Mangasarian, Hyuk-Joon Oh, Sree R. K. R. Mallina, and Kyoung-Mi Lee. The outstanding cooperation of the University of Wisconsin Surgical Pathology group, including Drs. Kennedy Gilchrist, Reza Hafez, Tom Warner, and Andres Friedl, is gratefully acknowledged.

\section{REFERENCES}

1. Fitzgibbons PL, Page DL, Weaver D, et al: Prognostic factors in breast cancer-College of American Pathologists Consensus Statement 1999. Arch Pathol Lab Med 124:966-978, 2000

2. Street WN, Wolberg WH, Mangasarian OL: Nuclear feature extraction for breast tumor diagnosis. Proc IS\&T/SPIE Int Symp Elec Imag 1905:861-870, 1993

3. Wolberg WH, Street WN, Mangasarian OL: Importance of nuclear morphology in breast cancer prognosis. Clin Cancer Res 5:3542-3548, 1999

4. Wolberg WH, Street WN, Mangasarian OL: Computer-derived nuclear features compared with axillary lymph node status for breast carcinoma prognosis. Cancer (Cancer Cytopathol) 81:172-179, 1997

5. Adair F, Berg J, Joubert L, et al: Long-term follow-up of breast cancer patients: The 30-year report. Cancer 33:1145-1150, 1974

6. Langlands AO, Pocock SJ, Ken GR, et al: Long-term survival of patients with breast cancer: A study of the curability of the disease. $\mathrm{Br}$ Med J 2:1247-1251, 1979

7. Kerr GR, Kunkler IH, Langlands AO, et al: (In)curability of breast cancer-A 30-year report of a series of 3933 cases. Breast 7:90-94, 1998

8. Street WN: Xcyt: A system for remote cytological diagnosis and prognosis of breast cancer, in Jain LC (ed): Soft Computing Techniques in Breast Cancer Prognosis and Diagnosis. Singapore, World Scientific Publishing, 2000, pp 297-322

9. Mandelbrot BB: The Fractal Geometry of Nature. New York, NY, Freeman, 1977

10. SPSS for Windows: Base System, Release 7.5. Chicago, IL, SPSS Inc., 1997

11. SPSS Advanced Statistics 7.5. Chicago, IL, SPSS Inc., 1997

12. Kaplan EL, Meier P: Nonparametric estimation from incomplete observations. J Am Stat Assoc 53:457-481, 1958

13. Baak JPA, VanDiest PJ, Stroet-Van Galen C, et al: Data processing and analysis in the multicenter morphometric mammary carcinoma project. Pathol Res Pract 185:657-663, 1989

14. Bloom H, Richardson W: Histological grading and prognosis in breast cancer. Br J Cancer 19:359-377, 1957

15. Boiesen P, Bendahl PO, Anagnostaki L, et al: Histologic grading in breast cancer-Reproducibility between seven pathologic departments. Acta Oncol 39:41-45, 2000

16. American Joint Committee on Cancer: Breast cancer staging, in American Joint Committee on Cancer: Manual for Staging of Cancer. Philadelhpia, PA, Lippincott Williams and Wilkins, 1982

17. Warmuth MA, Bowen G, Prosnitz LR, et al: Complications of axillary lymph node dissection for carcinoma of the breast: A report based on a patient survey. Cancer 83:1362-1368, 1998

18. Giuliano AE, Dale PS, Turner RR, et al: Improved axillary staging of breast cancer with sentinel lymphadenectomy. Ann Surg 222:394-399, 1995

19. Krag D, Weaver D, Ashikaga T, et al: The sentinel node in breast cancer: A multicenter validation study. N Engl J Med 339:941946, 1998

20. Baak JPA, Kurver PHJ, Snoo-Niewlaat AJE, et al: Prognostic indicators in breast cancer: Morphometric methods. Histopathology 6:327-339, 1982

21. Baak JPA, VanDop H, Kurver PHJ, et al: The value of morphometry to classic prognosticators in breast cancer. Cancer 56:374 382,1985

22. Stenkvist B, Westman-Naeser S, Vegelius J, et al: Analysis of reproducibility of subjective grading systems for breast carcinoma. J Clin Pathol 32:979-985, 1979

23. Wittekind C, Schulte E: Computerized morphometric image analysis of cytologic nuclear parameters in breast cancer. Anal Quant Cytol Histol 9:480-484, 1987

24. Fregene TA, Khanuja PS, George J, et al: Nuclear morphometry predicts for disease recurrence in early stage breast cancer [meeting abstract]. Proc Ann Meet Am Assoc Cancer Res 35:A1354, 1994

25. Eskelinen MJ, Pajarinen P, Collan Y, et al: Relationship between DNA ploidy and survival in patients with primary breast cancer. Br J Surg 76:830-834, 1989

26. Dressler LG, Seamer LC, Owens MA, et al: DNA flow cytom- 
etry and prognostic factors in 1331 frozen breast cancer specimens. Cancer 61:420-427, 1988

27. Fallenius AG, Auer GU, Carstensen JM: Prognostic significance of DNA measurements in 409 consecutive breast cancer patients. Cancer 62:331-341, 1988

28. Kallioniemi OP, Blanco G, Alavaikko M, et al: Improving the prognostic value of DNA flow cytometry in breast cancer by combining DNA index and S-phase fraction. Cancer 62:2183-2190, 1988

29. Sundquist M, Thorstenson S, Brudin L, et al: A comparison between flow cytometric assessment of S-phase fraction and Nottingham histologic grade as prognostic instruments in breast cancer. Breast Cancer Res Treat 63:11-15, 2000

30. Donegan WL: Tumor-related prognostic factors for breast cancer. CA Cancer J Clin 47:28-51, 1997
31. Tormey DC, Taylor SGI, Olson JE, et al: Adjuvant systemic therapy in premenopausal (CMF, CMFP, CMFPT) and postmenopausal (observation, CMFP, CMFPT) women with node-positive breast cancer, in Salmon SS (ed): Adjuvant Therapy of Cancer, Vo IV. Philadelphia, PA, Lippincott Williams and Wilkins, 1984, pp 359-368

32. Cummings FJ, Gray R, Davis TE, et al: Adjuvant tamoxifen treatment of elderly women with stage II breast cancer: A double-blind comparison with placebo. Ann Int Med 103:324-329, 1985

33. Taylor SG IV, Kuniman MW, Sleeper LA, et al: Six-year results of the Eastern Cooperative Oncology Group trial of observation versus CMFP versus CMFPT in postmenopausal patients with node-positive breast cancer. J Clin Oncol 7:879-889, 1989 